
\title{
Research S Surate \\ Oleanolic acid inhibits the proliferation of Hela cells in cervical cancer by regulating the ACSL4 ferroptosis signaling pathway
}

\section{Xiaofei Jiang}

Xuzhou City Hospital of Chinese Medicine

Mingqing Shi

Lishui Hospital of Chinese Medicine

Miao Sui

Suzhou City Hospital of Chinese Medicien

Yizhen Yuan

The First Clinical Medicial College of Nanjing University of Chinese Medicine

Shuang Zhang

Zhangjiagang Hospital of Chinese Medicine

Qinhua Xia (Dxa-qh@163.com)

Jiangsu Provincial Hospital of Chinese Medicine

Kai Zhao

Xuzhou City Hospital of Chinese Medicine

Research

Keywords: Cervical cancer, ferroptosis, Oleanolic acid, ACSL4 RNAi

Posted Date: November 9th, 2020

DOI: https://doi.org/10.21203/rs.3.rs-102345/v1

License: (c) (1) This work is licensed under a Creative Commons Attribution 4.0 International License.

Read Full License 


\section{Abstract}

Background: Cervical cancer continues to be the leading cause of cancer deaths among women worldwide. Oleanolic acid (OA) is a naturally occurring substance found in the leaves, fruits, and rhizomes of plants that has anti-cancer activity.

Methods: We used tumor-bearing mice as the animal model and Hela cell as cell models. Western blot was used for detecting the expression of proteins in ferroptosis related proteins acyl-CoA synthase longchain family member 4 (ACSL4), ferritin heavy chain (FTH1), transferrin receptor (TfR1) and glutathione peroxidase 4 (GPX4) in vivo and in vitro. MTT and EdU was for the detection of the viability of Hela cells.

Results: In vivo experiments showed that OA significantly reduced the size and mass of cervical cancer tumors. In vitro experiments showed that $\mathrm{OA}$ significantly reduced the viability and proliferation capacity of Hela cells. In both in vivo and in vitro assays, $\mathrm{OA}$ increased the level of oxidative stress and $\mathrm{Fe}^{2+}$ content, and increased the expression of ferroptosis related proteins. We found high expression of ACSL4 in both xenograft models and cervical carcinoma cells. Meanwhile, knockdown of ACSL4 expression using shRNA in cervical cancer cells significantly increased cell viability and proliferation. In addition, decreased ROS levels and GPX4 were detected in ACSL4 knockdown cervical cancer cells, suggesting that ACSL4 inhibition may contribute to the reduction of ferroptosis within Hela cells and thus improve Hela cell survival.

Conclusion: Promotion of ACSL4 dependent ferroptosis through OA may be an effective approach to treat cervical cancer.

\section{Introduction}

Cervical cancer is one of the most common tumors in women and is the leading cause of cancerous death in women [1]. Most patients with cervical cancer receive radiation therapy and adjuvant chemotherapy, which can significantly improve patient survival [2]. However, excessive radioresistance, chemical resistance, and tumor metastasis pose problems for treatment, and the biology of cervical cancer and the underlying molecular mechanisms have not been proven. Therefore, exploring the signaling mechanisms will provide an effective reference for the treatment of cervical cancer.

Ferroptosis is a regulated form of cell death, which means it can be attenuated or accelerated by specific genetic or pharmacological manipulations [3]. With the increased understanding of ferroptosis, it has been found that ferroptosis plays an important role in cancers such as kidney and liver cancer, where it can inhibit tumor growth in vivo and kill tumor cells in vitro [4, 5]. Therefore, the induction of tumor ferroptosis by small molecules has become an available strategy for the treatment of cancer. In addition, the alterations of expression in tumor genes affect ferroptosis in tumor and many studies have demonstrated that the acyl-CoA synthase long chain family member 4 (ACSL4) gene regulates ferroptosis [6-8]. Yuan et al., 2016 [6]demonstrate that ACSL4 contributes to the accumulation of lipid intermediates during hypertrophy disease, and oxidative stress is essential for ferroptosis. 
Oleanolic acid (OA) is a pentacyclic triterpene naturally distributed in the leaves, fruits and seeds of plants [9]. OA has been shown to have several biological activities, such as antioxidant [10], antiinflammatory [11], and antitumor activities [12]. Although the antitumor activity of OA has been reported, its specific role in cervical cancer is unknown.

In this study, we hypothesized that OA would not only reduce Hela tumor invasion in mice, but also inhibit Hela cell growth and proliferation. We examined the regulation of oxidative stress, $\mathrm{Fe}^{2+}$ levels and ferroptosis related proteins. In addition, we examined the changes in the survival and proliferation capacity of human cervical cancer cells and the expression of ferroptosis related proteins after ACSL4 inhibition.

\section{Materials And Methods}

\section{Cell lines, mice and reagents}

Hela cell lines (human cervical cancer cell line) obtained from Nanjing University of Chinese Medicine were cultured in Dulbecco's Modified Eagle's Medium (DEME, Gibco, USA) with $10 \%$ fetal bovine serum (FBS, Gibco, USA) plus antibiotics ( $100 \mu \mathrm{g} / \mathrm{mL}$ penicillin-streptomycin, Beyotime, China) maintained at $37^{\circ} \mathrm{C}$ in a humidified atmosphere $(90 \%)$ containing $5 \% \mathrm{CO}_{2}$. Male BALB/c Nude mice $(20 \pm 2 \mathrm{~g}, 5$ weeks old) supplied by Hangzhou Ziyuan Experimental Animal Technology Co. LTD (SYXK-20180049) were used in this study. The 3-(4, 5-dimethylthiazol-2-yl)-2, 5-diphenyl tetrazolium (MTT) was purchased from Sigma-Aldrich company (USA). Oleanolic acid (OA, purity $\geq 98 \%$ ) from Solarbio (08260, China) was dissolved in 50\% DMSO (Sigma, USA) and diluted with double distilled water to make the final concentrations of DMSO less than $0.1 \%$ to reduce cytotoxicity [13].

\section{Animal modeling and grouping}

The animal experiments were conducted in accordance with the accepted standards of animal care. This study was approved by the Xuzhou City Hospital of Chinese Medicine Animal Care and Use Committee. The nude mice were kept under specefic pathogen free (SPF) conditions for one week, and $5 \times 10^{7}$ Hela cells were injected under the left axillary skin after acclimatization. The tumors of Hela cell-inoculated mice were measured every 3 days after modeling, and tumor diameter $\geq 0.5 \mathrm{~cm}$ was considered successful. The tumor-bearing mice were randomly divided into control group $(\mathrm{n}=6), 40 \mathrm{mg} / \mathrm{kg}$ OA group $(n=6)$ and $80 \mathrm{mg} / \mathrm{kg}$ OA group $(n=6)$. All groups underwent subcutaneous injection modeling, and control mice received saline intraperitoneal injections, while $40 \mathrm{mg} / \mathrm{kg}$ OA group and $80 \mathrm{mg} / \mathrm{kg}$ OA group received $40 \mathrm{mg} / \mathrm{kg}$ oleanolic acid and $80 \mathrm{mg} / \mathrm{kg}$ oleanolic acid intraperitoneal lasted 15 days. Tumors were measured and weighed every 3 days, and tumor volume $\left(\mathrm{V} ; \mathrm{mm}^{3}\right)$ was calculated according to the following formula: $V=$ Major axis $\times$ Minor axis $\times$ height $\times 0.52$. After disposing of nude mice, tumors were removed and weighed. 
The tumors were fixed in $4 \%$ paraformaldehyde solution for 24 hours, dehydrated, embedded in paraffin, and then serially sectioned $(4 \mu \mathrm{m})$ with a microtome (Histocore Biocut, Thermo Fisher Scientific, USA). The samples were then stained with hematoxylin and eosin (HE) and then dehydrated twice. Sections were sealed with glass and placed under a microscope (Olympus, Japan) to observe the morphology of the cells.

\section{Determination of iron content, MDA and GSH}

The iron content of the different groups was analyzed using an iron assay kit (ab83366, Abcam, UK) according to the manufacturer's instructions. The absorbance was measured at $593 \mathrm{~nm}$ using a micro spectrophotometer (Nanodrop, Thermo). Oxygen radicals act on the unsaturated fatty acids of lipids to produce lipid peroxide; the latter is gradually broken down into a complex series of compounds, such as malondialdehyde (MDA), so oxidation can be detected by measuring the level of MDA. The content of MDA was measured using Malondialdehyde (MDA) content detection kit (BC0025, Solarbio, China) and the absorbance of the supernatant was determined at $532 \mathrm{~nm}$. Reduced glutathione (GSH) is the main antioxidant sulfhydryl substance in cells, and plays an important role in antioxidation, protein sulfhydryl protection and amino acid transport across membranes. The Micro Reduced Glutathione (GSH) Assay Kit (BC1175, Solarbio, China) was used to measure the absorbance at $412 \mathrm{~nm}$ to detect the GSH activity in samples.

Determination of reactive oxygen species (ROS) in vivo and in vitro

Tumor tissues were rapidly frozen and sectioned, and sections were incubated in $5 \mu \mathrm{M}$ ROS fluorescent probes dihydroethidium (DHE, D1008, UE, China) solution at $37^{\circ} \mathrm{C}$ for $30 \mathrm{~min}$ and washed three times with PBS for 5 min each time. ROS-positive cells showed red under fluorescence microscopy. Statistical analysis of fluorescence intensity was performed using imageJ software. The treated cells were incubated in DEME containing 50 MM C11 BODIPY 581/591 Lipid Peroxidation Sensor (GC40165, GLPBIO, USA) solution at $37^{\circ} \mathrm{C}$ for $1 \mathrm{~h}$. The cells were washed three times and analyzed by flow cytometry (Beckman Coulter, USA).

\section{Western blot analysis}

The tumor and Hela cell lysates were prepared, washed in cold phosphate buffer saline (PBS), resuspended in a lysis buffer and sonicated the lysate. Equal amounts of protein ( $50 \mu \mathrm{g} / \mathrm{lane})$ were electrophoresed in SDS-PAGE gels and transferred to a PVDF membrane (Millipore). ACSL4 (ab155282, Abcam, UK), ferritin heavy chain (FTH1, 3998, CST, USA), glutathione peroxidase 4 (GPX4, ab125066, Abcam, UK) and transferrin receptor (TfR1, ab1086, Abcam, UK) primary antibodies were used. HRPconjugated Goat Anti-Mouse IgG ( $\mathrm{H}+\mathrm{L})$ HRP (1:5000, No. S0002, Affinity) or Goat Anti-Rabbit IgG $(\mathrm{H}+\mathrm{L})$ $\operatorname{HRP}(1: 5000$, No. S0001, Affinity) was used as the secondary antibody. The protein bands were scanned by a gel analysis system and the grayscale values of the strips were analyzed using image analysis software (Image $J$ ). 
Hela cells were seeded overnight, and then incubated with various concentrations of OA $(5 \otimes 10 \otimes 20 \mu \mathrm{M})$ for $48 \mathrm{~h}$. For each concentration, a control sample was maintained in the untreated state and received an equal volume of medium. All different treatments were performed in triplicate.

\section{Cell viability and proliferation assays}

Cell viability was determined by the MTT method. The cells were spread in a 96-well plate with 5000 cells per well and allowed to grow for 24 hours and then treated with OA $(5,10$ and $20 \mu \mathrm{M})$ for 48 hours. MTT solution ( $5 \mathrm{mg} / \mathrm{mL}$, in PBS) was used to label cells for $2 \mathrm{~h}$ and then dissolved with DMSO $(100 \mu \mathrm{L})$ for the fumazen dye it produced. The absorbance was measured at $570 \mathrm{~nm}$ in an ELISA plate reader [14].

The Hela cells were spread in 48-well plates (5000 cells/well) and cultured for $24 \mathrm{~h}$. After $24 \mathrm{~h}$, the cells were treated with different concentrations of OA $(5,10$ and $20 \mu \mathrm{M})$ and incubated for $48 \mathrm{~h}$. The medium containing EdU (EdU apollo 567in vitro kit, Solarbio, China) was replaced and incubated for $3 \mathrm{~h}$. After incubation, the cells were fixed and stained according to the manufacturer's instructions. The positivity of EdU was recorded based on the combined images of EdU and Hoechst33342.

shRNA interference silences the ACSL 4 gene

Lentivirus particles carrying si-ACSL4 and the empty vectors were constructed by Shanghai Genechem Co., Ltd. Transfections were performed with Lentivirus Transduction System according the manufacturer's instructions. The Lentivirus vectors were co-cultured with Hela cells to facilitate transfection. The group of Hela cells transfected with empty vector was used as the control group.

\section{Statistical analysis}

The statistics were performed using GraphPad Prism 8 . All data are presented as mean \pm standard deviation (SD) of three independent experiments. T-test was used to compare the data between the two groups, ANOVA was used to compare data among three groups. Results were considered to be statistically significant for values * $P<0.05, * * P<0.01$.

\section{Results}

OA suppresses the growth of tumor

The chemical structural formula of OA is shown in Figure $1 \mathrm{~A}$. To verify whether OA could be used as a potential drug for cervical cancer, we tested the effect of inhibition of OA on cervical cancer tumor growth using a mouse xenograft model. Intraperitoneal injection-treated nude mice in $40 \mathrm{mg} / \mathrm{kg}$ or $80 \mathrm{mg} / \mathrm{kg} \mathrm{OA}$ groups formed smaller tumors compared to the control group (Figure 1B). Moreover, the volume and mass of tumor were also significantly reduced $(p<0.05$, Figure $1 \mathrm{C}$ and $\mathrm{D})$. As shown in Figure 1E, tumor pathology sections of $\mathrm{HE}$ verified that $\mathrm{OA}$ treatment of tumor-bearing mice resulted in massive infiltration 
of tumor cells. Overall, these findings confirmed that OA has the ability to suppress cervical cancer tumors.

\section{$O A$ increases the accumulation of iron and ROS in tumor}

$\mathrm{Fe}^{2+}$ was significantly accumulated in the tumor tissues of OA-treated tumor-bearing mice compared to the control group (Figure 2A). In Figure 2B, the MDA activities of $40 \mathrm{mg} / \mathrm{kg} O A$ and $80 \mathrm{mg} / \mathrm{kg}$ OA groups were higher than that of control group significantly $(p<0.05)$. On the contrary, the GSH contents in the 40 $\mathrm{mg} / \mathrm{kg} O A$ and $80 \mathrm{mg} / \mathrm{kg}$ OA groups were lower than that in the control group observably $(p<0.05$, Figure $2 \mathrm{C})$. Since lipid peroxidation is a feature of rust disease, we next measured ROS content in tumor tissues [15]. The brightness of the ROS-DHE probe was significantly higher in the $40 \mathrm{mg} / \mathrm{kg} O A$ and $80 \mathrm{mg} / \mathrm{kg} \mathrm{OA}$ groups than in the control group ( $p<0.05$, Figure $2 \mathrm{D}$ and $\mathrm{E})$.

\section{OA regulates the expression of ferroptosis related proteins in tumor}

As several studies have reported that ferroptosis regulates tumors, experiments were performed to test whether OA could mediate the ferroptosis signaling pathway. For this purpose, the translation levels of key parameters in the ferroptosis signaling pathway were analyzed (Figure $3 \mathrm{~A}$ and B). Under OA-treated conditions, the expressions of ACSL4 and TfR1 were stronger in the $80 \mathrm{mg} / \mathrm{kg}$ group than that in the 40 $\mathrm{mg} / \mathrm{kg}$ group, whereas the trend was reversed for FTH1 and GPX4. Similarly, the expression levels of these proteins in Hela cells showed the same trend as in tumors (Figure $5 \mathrm{C}$ and D).

OA treatment inhibits the viability of Hela cell and increases the accumulation of iron and ROS

After treatment with $\mathrm{OA}(0,5,10,20 \mu \mathrm{M})$, the viability of Hela cells decreased with increasing concentration of $\mathrm{OA}$ (Figure $4 \mathrm{~A}$ ). The contents of $\mathrm{Fe}^{2+}$ and MDA increased with increasing OA concentration, while GSH decreased with increasing OA concentration (Figure 4B-D). At the same time, the content of intracellular ROS increased with the increase of OA concentration (Figure 4E). These results indicated that the addition of OA coincubation decreased Hela cell viability and increased the level of intracellular oxidative stress.

\section{OA inhibits Hela cell proliferation in a dose-dependent manner}

To elucidate the effect of $O A$ on cell proliferation, Hela cells were treated with different concentrations of $\mathrm{OA}(0,5,10$ and $20 \mu \mathrm{M})$ and subjected to EdU and Hoechst33342 assays (Fig. 5A) and analyzed (Fig. 5B). EdU can be doped into the DNA of proliferating cells, and after capturing images with fluorescence microscopy, the total number of EdU cells was counted and expressed relative to the EdU cell counts of Hoechst33342 cells. The proliferative capacity of Hela cells decreased significantly with increasing OA concentration ( $p<0.05$, Fig. $5 A$ and $B)$.

Silencing of ACSL 4 decreases the sensitivity of Hela cells to $O A$ 
As shown in Figure 6A, si-ACSL4 successfully blocked the expression of ACSL4. In Figure 6B, cell viability increased after ACSL4 was inhibited. At the same time, the proportion of newly proliferating cells was down-regulated as seen by EdU proliferation analysis (Fig. 6C and D), and the intracellular ROS content was also down-regulated by flow cytometry analysis (Fig. 6E). ACSL4 knockdown reduced OA-induced ACSL4 high expression and significantly increased OA-induced GPX4 expression (Figure $6 \mathrm{~F}$ and $\mathrm{G}$ ). The results suggest that the OA-induced ferroptosis in Hela cells can be reduced by inhibiting the expression of ACSL4.

\section{Discussion}

Cervical cancer is one of the most common tumors in women and is clinically treated with radiotherapy and chemotherapy. However, the use of these methods is limited due to metastasis and resistance of the tumor. Therefore, exploring the signaling mechanisms of cervical cancer will provide an effective reference for treatment. Abnormal accumulation of iron, ROS or both is associated with various diseases of cancer. The induction of ferroptosis is an approach to suppressing tumor growth [16, 17], but oxidative stress-induced ferroptosis has not yet been linked to the pathology of cervical cancer. In this study, we elucidated the regulatory role of ACSL4 in ferroptosis and its mechanisms in cervical cancer cells. Namely, OA increased intracellular ROS levels by regulating expressions of ferroptpsis related proteins, which lead to ferroptosis. Further si-ACSL4 experiments showed that ferroptosis was ameliorated after ACSL4 was silenced. These new findings are shown in Figure 7.

Ferrroptosis is an iron-dependent form of cell death caused primarily by the peroxidation of phospholipids (PLs) containing polyunsaturated fatty acids (PUFAs) [8]. ACSL4 is a long-chain fatty acidcoenzyme A ligase that is required for PUFA-PLs biosynthesis, which in turn lead to elevated lipid peroxidation and ferroptosis $[18,19]$. Yuan et al. 2016 [6] found that ACSL4 contributes to the accumulation of lipid intermediates during ferroptosis and identified ACSL4 as a biomarker and contributor to ferroptosis. $\mathrm{OA}$ is an excellent natural anticancer agent, and recent studies have shown that OA can inhibit the growth of leukemia, non-small cell lung cancer (NSCLC) cell lines and other malignant tumors [20-22] . In both in vivo and in vitro experiments, results showed that ACSL4 expression levels and ROS accumulation were further increased were further increased in OA-treated tumor tissues and Hela cells, indicating an increase in the intensity of ferroptosis. GPX4 could use GSH to convert lipid peroxides (PUFA-PL-OOH) into non-toxic lipid alcohol (PUFA-PL-OH), which removes the accumulation of lipid peroxides in cells, thereby reducing lipid peroxidation and inhibiting ferroptosis [8, 23].

Our results also suggested that inhibition of ACSL4 reduces the occurrence of ferroptosis. Since ACSL4 regulates lipid peroxidation, suppression of ACSL4 limits erastininduced lipotoxicity in ferroptosis, which is consistent with previous studies $[24,25]$. The process of ferroptosis is dynamic and involves iron metabolism in addition to lipid peroxidation [17]. Iron, which can alternate between the insoluble oxidized $\mathrm{Fe}^{3+}$ form and the reduced $\mathrm{Fe}^{2+}$ pro-oxidant form, can induce membrane lipid peroxidation by generating soluble free radicals, highly reactive oxygen species (ROS) and hydroxide ions $\left(\mathrm{OH}^{-}\right)$through the Fenton reaction [26]. The transferrin ( $\mathrm{Tf}$ ) pathway is important for the acquisition of iron by cells, and Tf-Fe ${ }^{3+}$ 
delivers iron to cells by binding to TfR1 on the cell surface and is released as $\mathrm{Fe}^{2+}$ via divalent metal transporter 1 (DMT1) [27]. Ferritin is the major intracellular iron storage protein, containing both ferritin heavy chain (FTH1) and ferritin light chain (FTL1). However, only FTH1 is able to convert Fe ${ }^{2+}$ to $\mathrm{Fe}^{3+}$ for storage in the ferritin mineral nucleus through its ferritin peroxidase activity to prevent Fenton reactions from occurring [28]. In our study, as ACSL4 levels increased, TfR1 expression significantly increased and FTH1 expression decreased. OA can effectively upregulated ferroptosis by promoting the expression ACSL4. After silencing of ACSL4, Hela cells decreased their sensitivity to OA. Thus, OA is targeted to promote ACSL4-mediated ferroptosis, thereby inhibiting the growth and proliferation of cervical cancer cells.

\section{Conclusion}

$\mathrm{OA}$ is a natural activator of ACSL4 and can modulate multiple levels and targets simultaneously. Notably, ACSL4-mediated ferroptosis activation reduced the size of cervical cancer tumors, decreased the viability of Hela cells, and enhanced their anticancer effects in cervical cancer. This supports that OA can be targeted to promote ACSL4 activation of ferroptosis as a potential anticancer agent in cervical cancer.

\section{Abbreviations}

ACSL4: acyl-CoA synthase long chain family member 4; DEME: Dulbecco's Modified Eagle's Medium; DHE: dihydroethidium; DMT1: divalent metal transporter 1; FBS: fetal bovine serum; FTH1: ferritin heavy chain; FTL1: ferritin light chain; GPX4: glutathione peroxidase 4; GSH: glutathione; HE: hematoxylin and eosin; MDA: malondialdehyde; MTT: 3-(4, 5-dimethylthiazol-2-yl)-2, 5-diphenyl tetrazolium; NSCLC: nonsmall cell lung cancer; OA: oleanolic acid; PBS: phosphate buffer saline; PLs: phospholipids; PUFAs: polyunsaturated fatty acids; ROS: reactive oxygen species; SD: standard deviation; SPF: specefic pathogen free; Tf: transferrin; TfR1: transferrin receptor;

\section{Declarations}

\section{Acknowledgements}

Not applicable.

\section{Authors' contributions}

JXF, SM and ZK performed the experiments and analyzed the data and and writing the manuscript; SMQ and $Y Y Z$ were the major contributor in designing the research. ZS provided guidance for the optimization of experiments. All authors read and approved the final manuscript.

\section{Funding}

This study was supported by The National Natural Science Foundation of China (Nos. 81473713). 


\section{Availability of data and materials}

The datasets used and/or analysed during the current study are available from the corresponding author on reasonable request.

\section{Ethics approval and consent to participate}

Not applicable.

\section{Consent for publication}

Not applicable.

\section{Competing Interest}

The authors report no conflicts of interest in this work.

\section{References}

[1] Crosbie EJ, Einstei MH, Franceschi S, Kitchener HC. Human papillomavirus and cervical cancer. Lancet 2013;382:889-899

https://doi.org/10.1016/S0140-6736(13)60022-7

[2] Shen SN, Zhang SB, Liu P, Wang JF, Du HY. Potential role of microRNAs in the treatment and diagnosis of cervical cancer. Cancer Genetics 2020;248-249:25-30.

https://doi.org/10.1016/j.cancergen.2020.09.003

[3] Galluzzi L, Vitale I, Aaronson SA, Abrams JM, Adam D, Agostinis P, Andrews DW. Molecular mechanisms of cell death: recommendations of the Nomenclature Committee on Cell Death 2018. Cell Death Differ. 2018;25:486-541.

https://doi.org/10.1038/s41418-017-0012-4

[4] Sun X, Ou Z, Chen R, Niu X, Chen D, Kang R, Tang D. Activation of the p62- Keap1-NRF2 pathway protects against ferroptosis in hepatocellular carcinoma cells. Hepatology 2016;63:173-184.

https://doi.org/10.1002/hep.28251

[5] Yu H, Guo P, Xie X, Wang Y, Chen G. Ferroptosis, a new form of cell death, and its relationships with tumourous diseases. J. Cell. Mol. Med. 2017;21:648-657.

https://doi.org/10.1111/jcmm.13008 
[6] Yuan H, Li XM, Zhang XY, Kang R, Tang DL. Identification of ACSL4 as a biomarker and contributor of ferroptosis. Biochem. Bioph. Res. Co. 2016;478(3):1338-1343.

https://doi.org/10.1016/j.bbrc.2016.08.124

[7] Dixon SJ, Winter GE, Musavi LS, Lee ED, Snijder B, Rebsamen M. Human Haploid Cell Genetics Reveals Roles for Lipid Metabolism Genes in Nonapoptotic Cell Death. ACS Chem. Biol. 2015;10:1604-1609.

https://doi.org/10.1021/acschembio.5b00245

[8] Doll S, Proneth B, Tyurina YY, Panzilius E, Kobayashi S, Ingold I, Irmler M, Beckers J, Aichler M, Walch A. ACSL4 dictates ferroptosis sensitivity by shaping cellular lipid composition. Nat. Chem. Biol. 2017;13:91-98.

https://doi.org/10.1038/nchembio.2239

[9] Pollier J, Goossens A. Oleanolic acid. Phytochemistry 2012;77:10-15.

https://doi.org/10.1016/j.phytochem.2011.12.022

[10] Zhang W, Feng J, Cheng B, Lu Q, Chen X. Oleanolic acid protects against oxidative stressinduced human umbilical vein endothelial cell injury by activating AKT/eNOS signaling. Mol. Med. Rep. 2018;18:3641-3648.

https://doi.org/10.3892/mmr.2018.9354

[11] Kashyap D, Sharma A, Tuli HS, Punia S, Sharma AK. Ursolic acid and oleanolic acid: pentacyclic terpenoids with promising anti-inflammatory activities. Recent Pat. Inflamm. Allergy Drug Discov. 2016;10:21-33.

https://doi.org/10.2174/1872213X10666160711143904

[12] Sanchez-Quesada C, Lopez-Biedma A, Gaforio JJ. Oleanolic acid, a compound present in grapes and olives, protects against genotoxicity in human mammary epithelial cells. Molecules 2015;20:1367013688.

https://doi.org/10.3390/molecules200813670

[13] Liu Y, Deng SJ, Zhang Z, Gu Y, Xia SN, Bao XY. 6-Gingerol attenuates microglia-mediated neuroinflammation and ischemic brain injuries through Akt-mTOR-STAT3 signaling pathway. Eur. J. Pharmacol. 2020;883:173294.

https://doi.org/10.1016/j.ejphar.2020.173294 
[14] Hosseini A, Shafiee-Nick R, Mousavi SH. Combination of Nigella sativa with Glycyrrhiza glabra and Zingiber officinale augments their protective effects on doxorubicin-induced toxicity in h9c2 cells. Iran J. Basic Med. Sci. 2014;17:993-1000.

[15] Wang Y, Quan F, Cao QH, Lin YT, Yue CX, Bi R. Quercetin alleviates acute kidney injury by inhibiting ferroptosis. J. Adv. Res. 2020.

https://doi.org/10.1016/j.jare.2020.07.007

[16] Shen Z, Song J, Yung BC, Zhou Z, Wu A, Chen X, Emerging strategies of cancer therapy based on ferroptosis. Adv. Mater. 2018;30:e1704007.

https://doi.org/10.1002/adma.201704007

[17] Xie Y, Hou W, Song X, Yu Y, Huang J, Sun X, Kang R, Tang D. Ferroptosis: process and function. Cell Death Differ. 2016;23:369e379.

https://doi.org/10.1038/cdd.2015.158

[18] Lei G, Zhang Y, Koppula P, Liu X., Zhang J, Lin SH, Gan B. The role of ferroptosis in ionizing radiationinduced cell death and tumor suppression. Cell Res. 2020;30:146-162.

https://doi.org/10.1038/s41422-019-0263-3

[19] Kagan VE, Mao G, Qu F, Angeli JPF, Doll S, Croix CS. Oxidized arachidonic and adrenic PEs navigate cells to ferroptosis. Nat. Chem. Biol. 2017;13:81-90.

https://doi.org/10.1038/nchembio.2238

[20] Chen Z, Huang KY, Ling Y, Goto M, Duan HQ, Tong XH, Lee KH. Discovery of an oleanolic acid/hederagenin-nitric oxide donor hybrid as an EGFR tyrosine kinase inhibitor for non-small-cell lung cancer. J. Nat. Prod. 2019;82:3065-3073.

https://doi.org/10.1021/acs.jnatprod.9b00659

[21] Silva AM, Alvarado HL, Abrego G, Martins-Gomes C, Garduno-Ramirez ML, Garcia ML, Souto EB. In vitro cytotoxicity of oleanolic/ursolic acids-loaded in PLGA nanoparticles in different cell lines.

Pharmaceutics 2019;11:362.

https://doi.org/10.3390/pharmaceutics11080362

[22] Takemura M, Endo S, Matsunaga T, Soda M, Zhao HT, El-Kabbani O, Hara A. Selective inhibition of the tumor marker aldo-keto reductase family member 1B10 by oleanolic acid. J. Nat. Prod. 2011;74:12011206. 
https://doi.org/10.1021/np200118q

[23] Yang WS, Kim KJ, Gaschler MM, Patel M, Shchepinov MS, Stockwell BR. Peroxidation of polyunsaturated fatty acids by lipoxygenases drives ferroptosis. Proc. Natl Acad. Sci. USA 2016;113:E4966-E4975 .

https://doi.org/10.1073/pnas.1603244113

[24] Dixon SJ, Lemberg KM, Lamprecht MR, Skouta R, Zaitsev EM, Gleason CE. Ferroptosis: An IronDependent Form of Nonapoptotic Cell Death. Cell 2012;149(5):1060-1072.

https://doi.org/10.1016/j.cell.2012.03.042

[25]Conrad M, Kagan VE, Bayir H, Pagnussat GC, Head B, Traber MG, Stockwell BR. Regulation of lipid peroxidation and ferroptosis in diverse species. Genes Dev. 2018;32:602-619.

https://doi.org/10.1101/gad.314674

[26] Hout ME, Santos LD, Hamaï A, Mehrpour M. A promising new approach to cancer therapy: Targeting iron metabolism in cancer stem cells. Semin Cancer Biol. 2018;53:125-138.

https://doi.org/10.1016/j.semcancer.2018.07.009

[27] Torti SV, Torti FM. Cellular iron metabolism in prognosis and therapy of breast cancer. Crit. Rev. Oncog. 2013;18(5):435-448.

https://doi.org/10.1615/CritRevOncog.2013007784

[28] Arosio P, Ingrassia R, Cavadini P. Ferritins: a family of molecules for iron storage, antioxidation and more. Biochim. Biophys. Acta, 2009;1790:589-599.

https://doi.org/10.1016/j.bbagen.2008.09.004

\section{Figures}


A

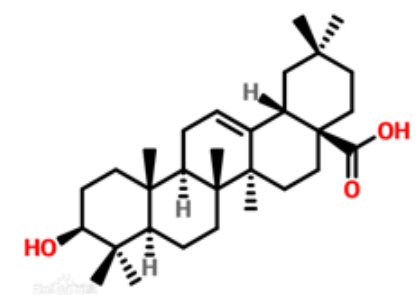

B

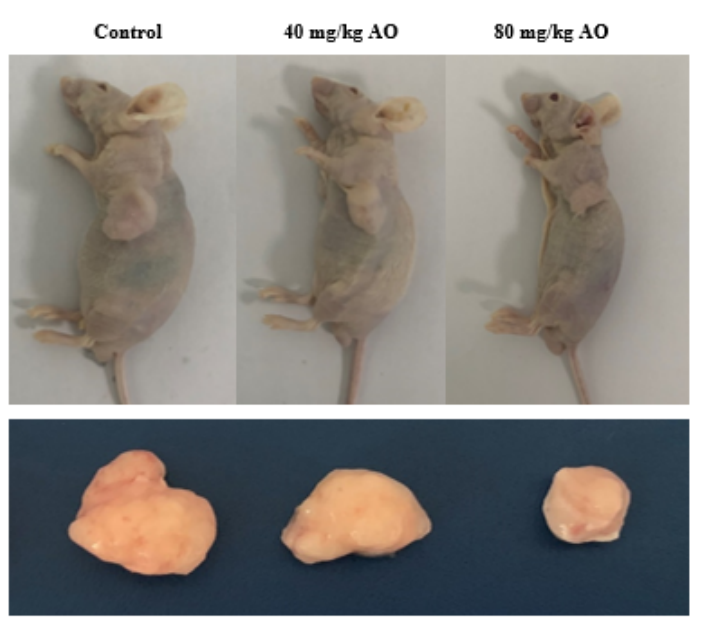

C

D
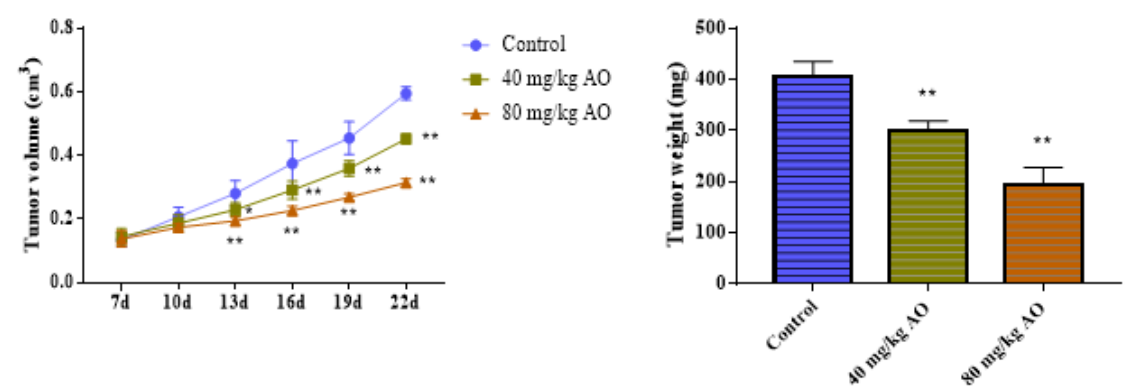

E

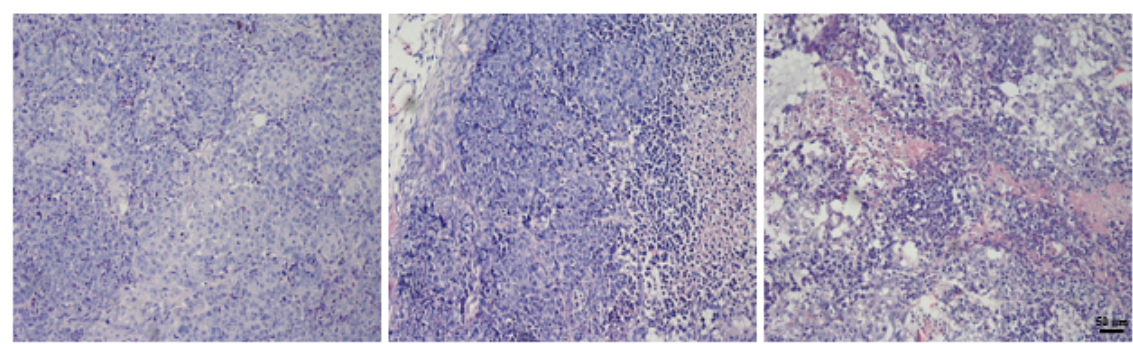

Figure 1

OA suppresses the growth of tumor. (A) The chemical structure of OA. (B) Figures of tumor masses. (C) OA inhibited the increase of tumor volume. (D) OA inhibited the increase of tumor weight. (E) HE staining of pathological sections of tumor. Compared with the control group, ${ }^{\star} \mathrm{P}<0.05,{ }^{*} \mathrm{P}<0.01$. Data are expressed as mean \pm SD $(n=3)$.

A

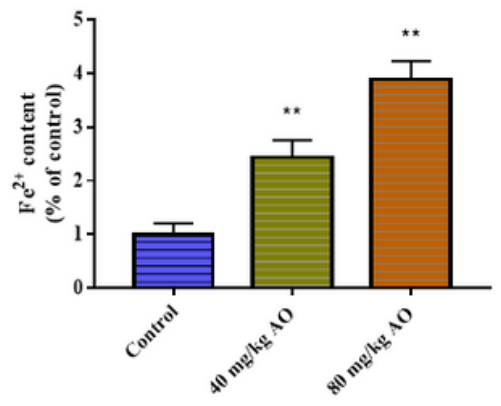

B

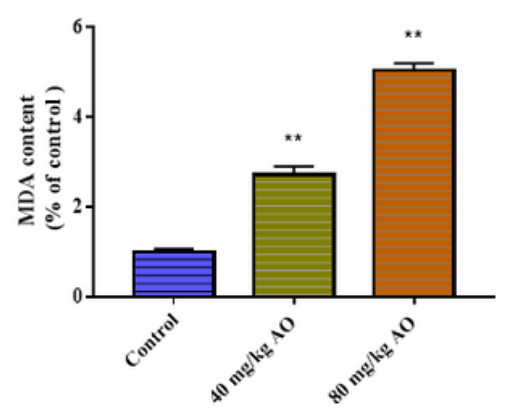

C

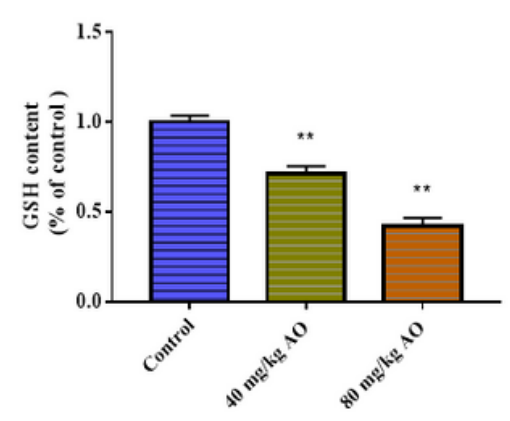

E

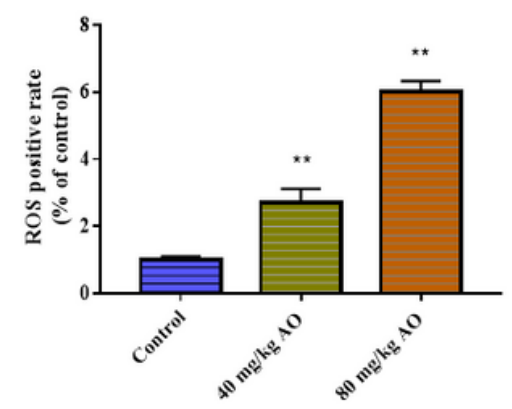

Figure 2 
OA increases the accumulation of iron and ROS in tumor. (A) The content of Fe2+ in tumor. (B) The content of MDA in tumor. (C) The content of GSH in tumor. (D) Pictures of ROS florescence in tumor. (E) Histogram of ROS in tumor. Compared with the control group, $* P<0.05,{ }^{*} \mathrm{P}<0.01$. Data are expressed as mean $\pm S D(n=3)$.

A

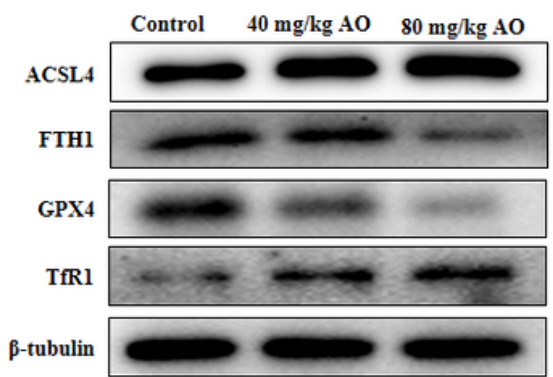

B
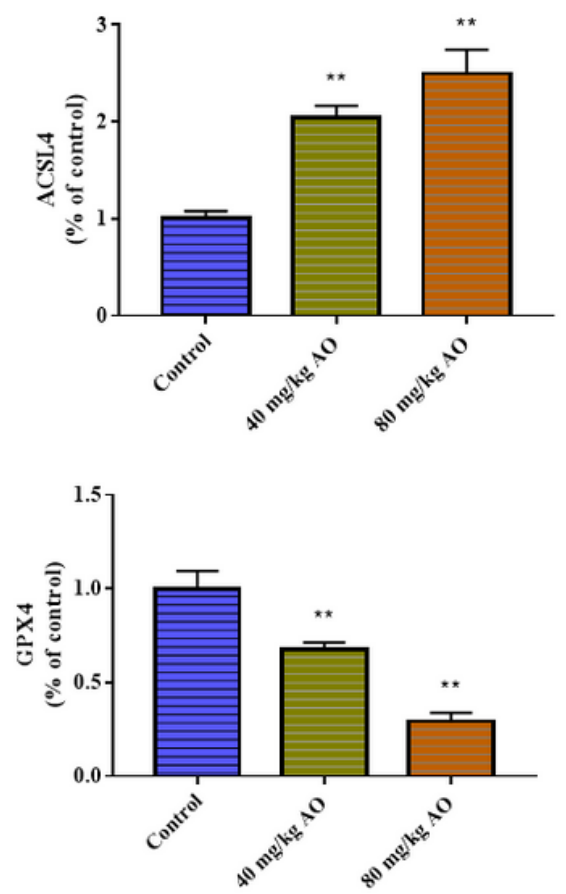
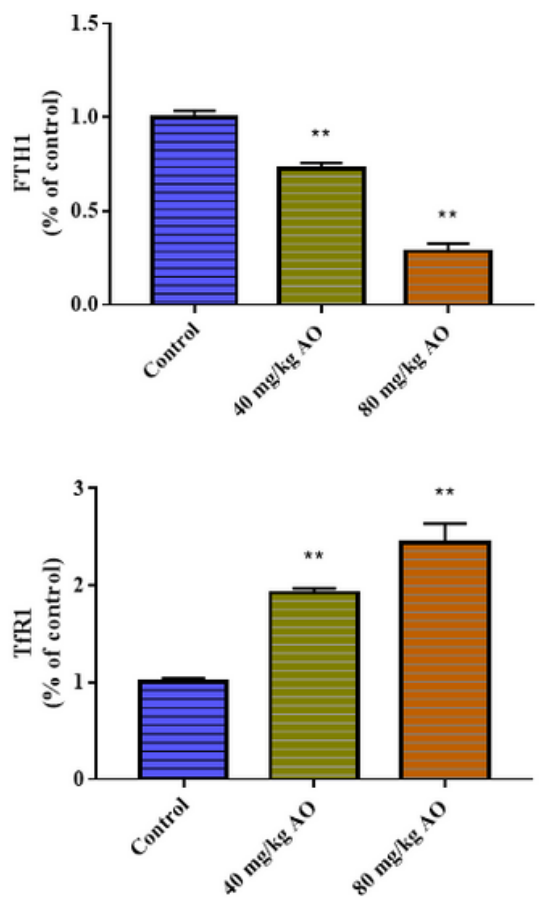

\section{Figure 3}

OA regulates the expression of ferroptosis related proteins in tumor. (A) Representative immunoblots of ferroptosis related proteins expressions in tumor lysates. (B) Administration of OA resulted in an increased ACSL4 and TfR 1 expression while FTH1 and GPX4 expression decreased in vivo. The density of bands was normalized to $\beta$-tubulin. Compared with the control group, $* P<0.05$, ${ }^{*} P<0.01$. Data are expressed as mean $\pm S D(n=3)$. 
A

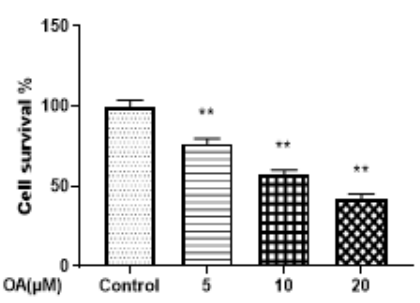

B

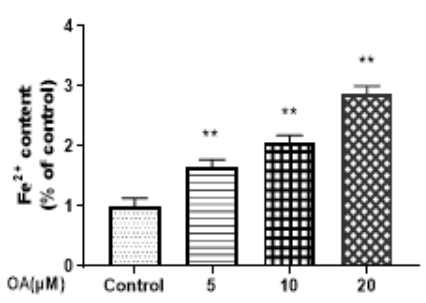

C

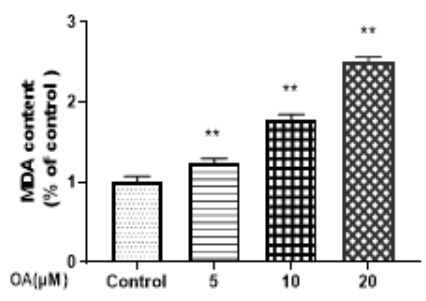

D

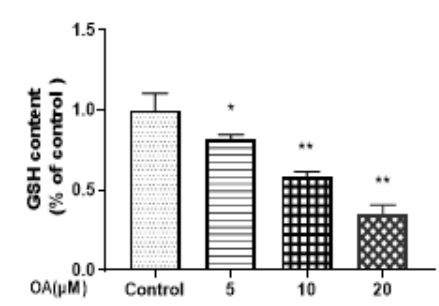

E
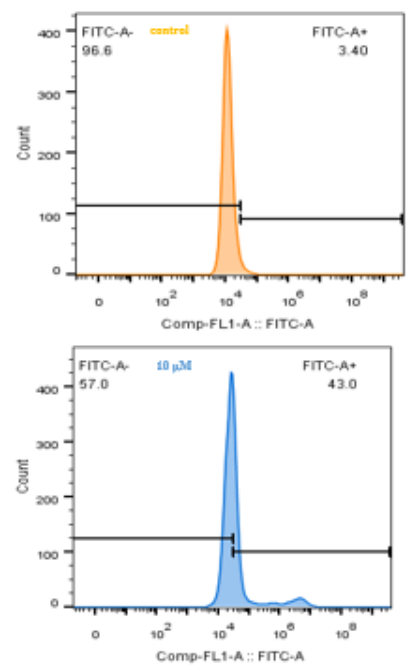
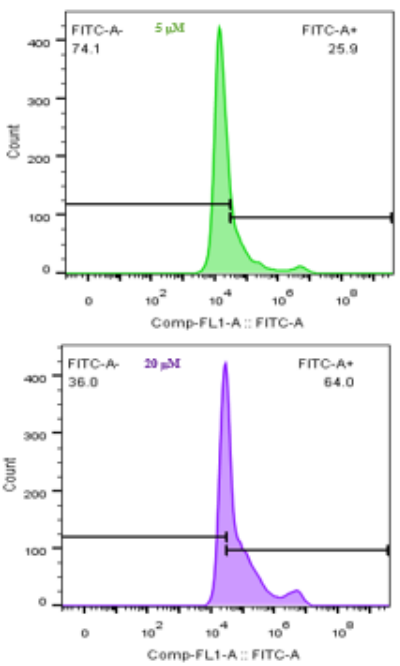

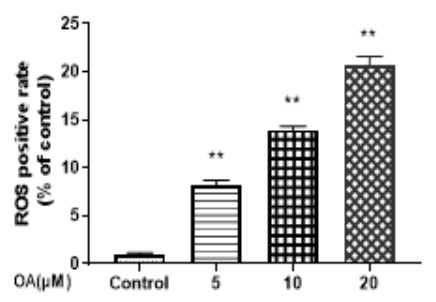

\section{Figure 4}

OA treatment inhibits the viability of Hela cell and increases the accumulation of iron and ROS. (A) The dose-dependent inhibitory effect of OA on Hela cells. (B) The content of Fe2+ in Hela cells. (C) The content of MDA in Hela cells. (D) The content of GSH in Hela cells. (E) The content of ROS in Hela cells. Compared with the control group, ${ }^{*} P<0.05, * \star P<0.01$. Data are expressed as mean $\pm S D(n=3)$. 
A
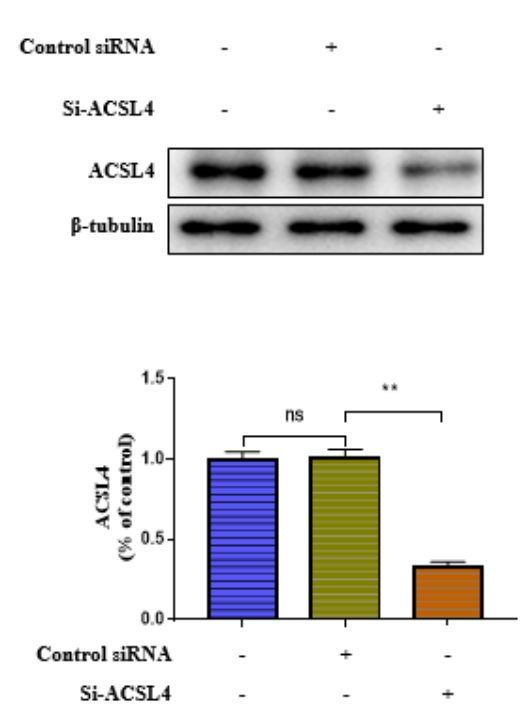

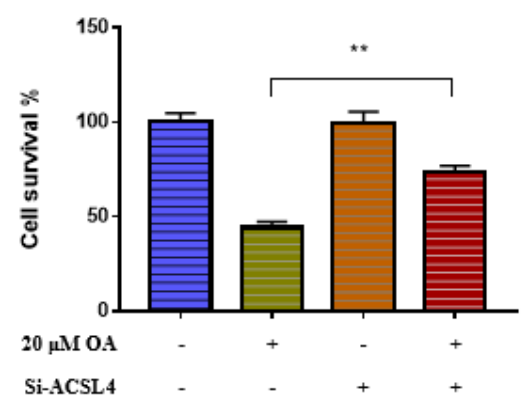

$\mathrm{C}$

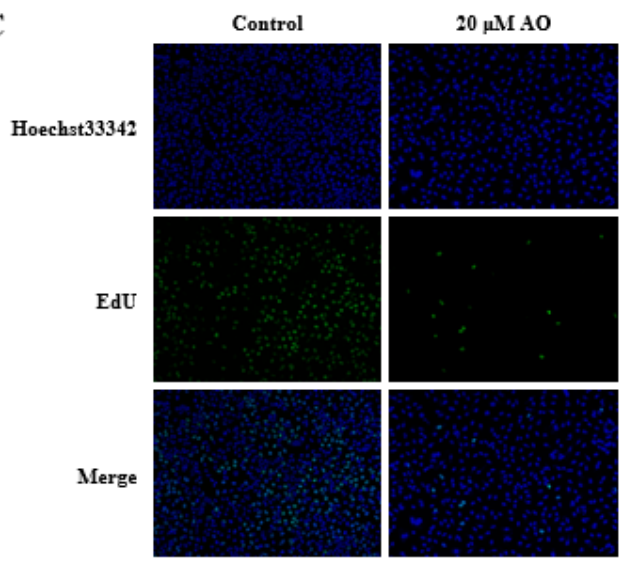

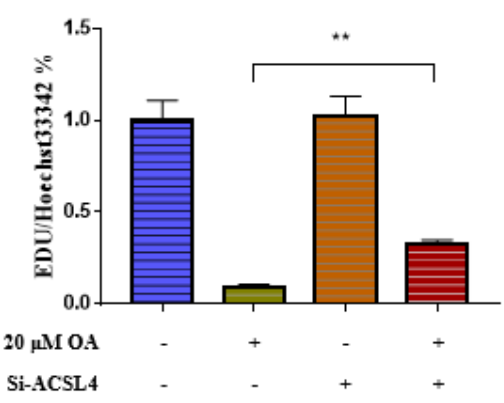

Si-ACSL4

Si-ACSL $4+20 \mu \mathrm{M}$ AO

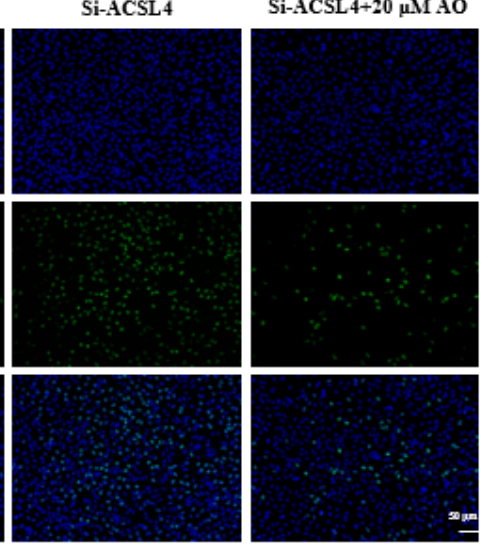

\section{Figure 5}

$\mathrm{OA}$ inhibits the proliferation and decreases the ferroptosis related proteins expressions in Hela cells. (A) Representative images of EdU and Hoechst33342 in Hela cells. (B) Proliferation efficiency of Hela cells. (C) Representative immunoblots of ferroptosis related proteins expressions in Hela cells. (D) Administration of OA resulted in an increased ACSL4 and TfR1 expression while FTH1 and GPX4 expression decreased in vitro. The density of bands was normalized to $\beta$-tubulin. Compared with the control group, ${ }^{\star} P<0.05,{ }^{\star} * P<0.01$. Data are expressed as mean $\pm S D(n=3)$. 
B

A
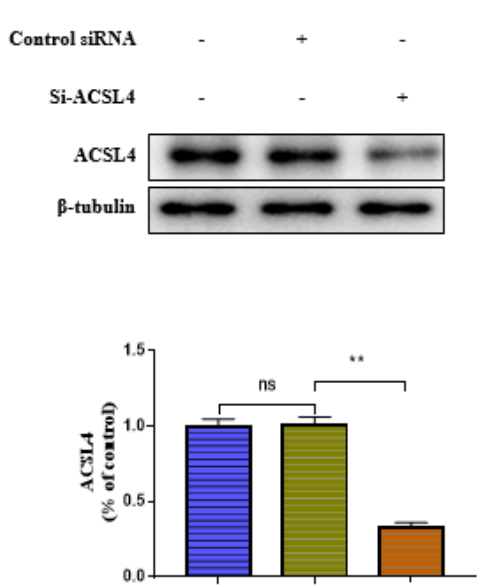

Control siRNA

Si-ACSL4

E
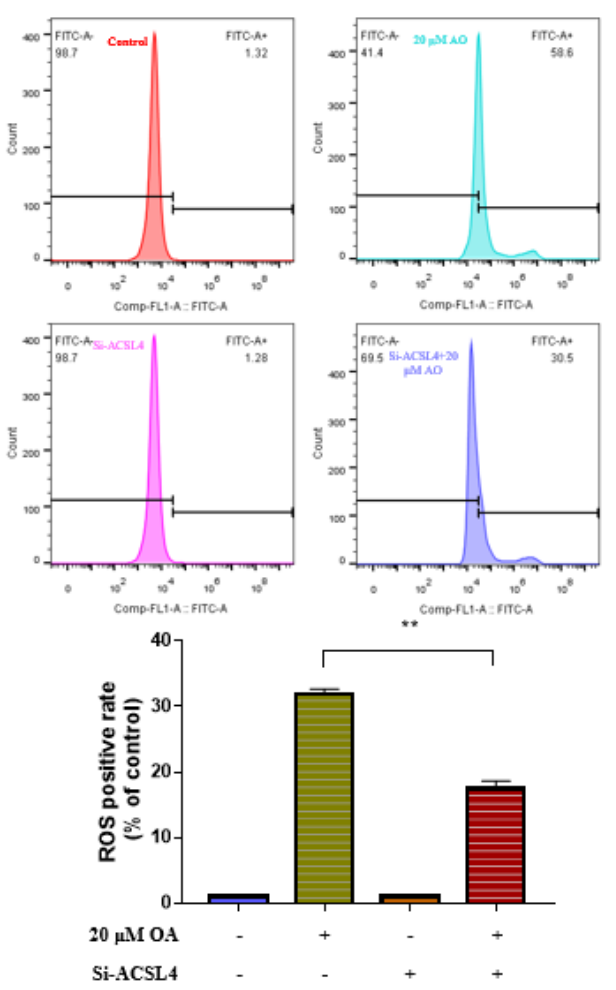

D
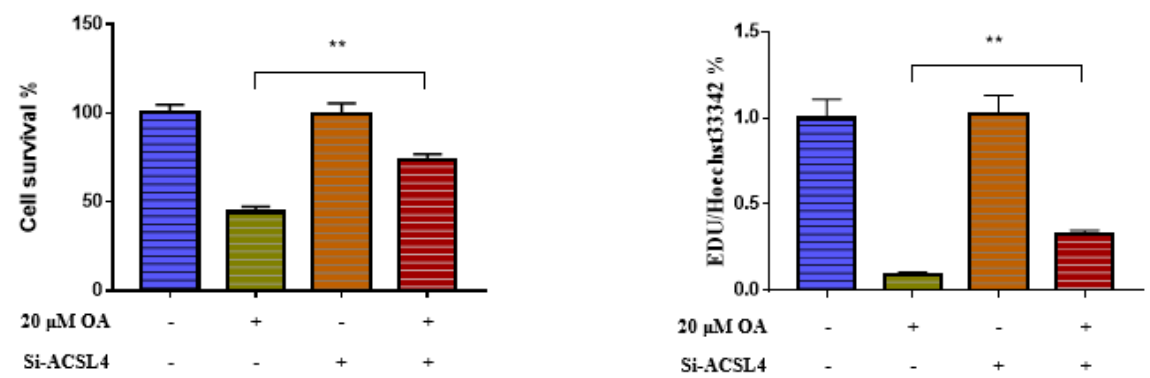

C

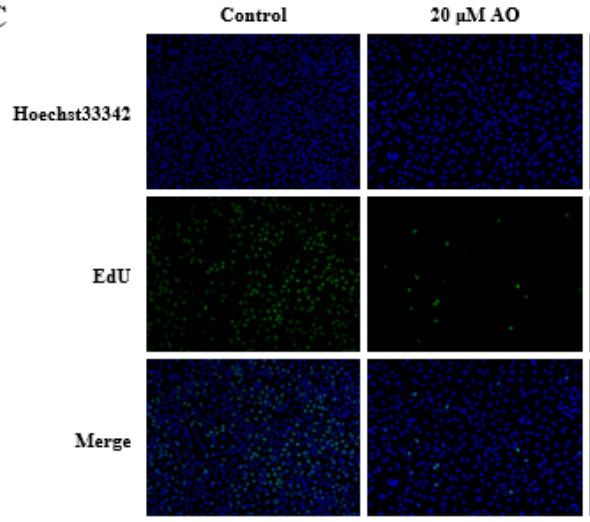

$20 \mu \mathrm{M}$ AO
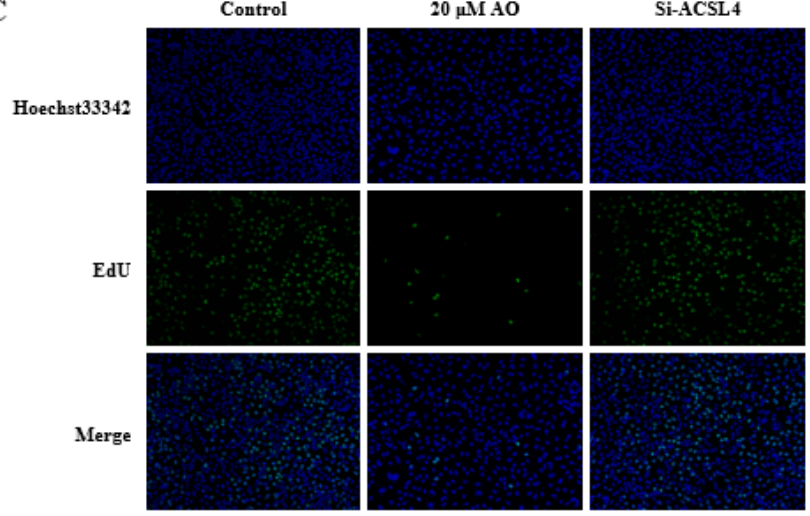

i-ACSL 4+20 $\mu \mathrm{M}$ AO

F
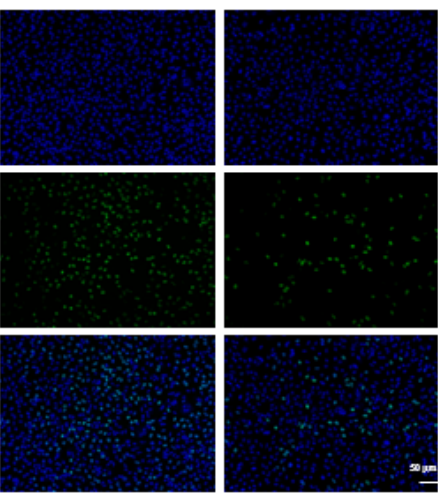

G

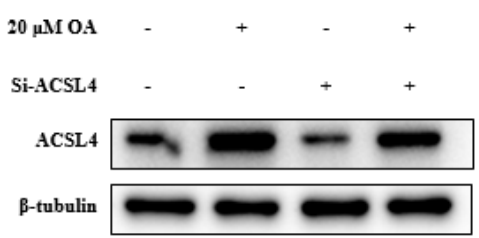

$20 \mu \mathrm{M} \mathrm{OA}$

Si-ACSL 4
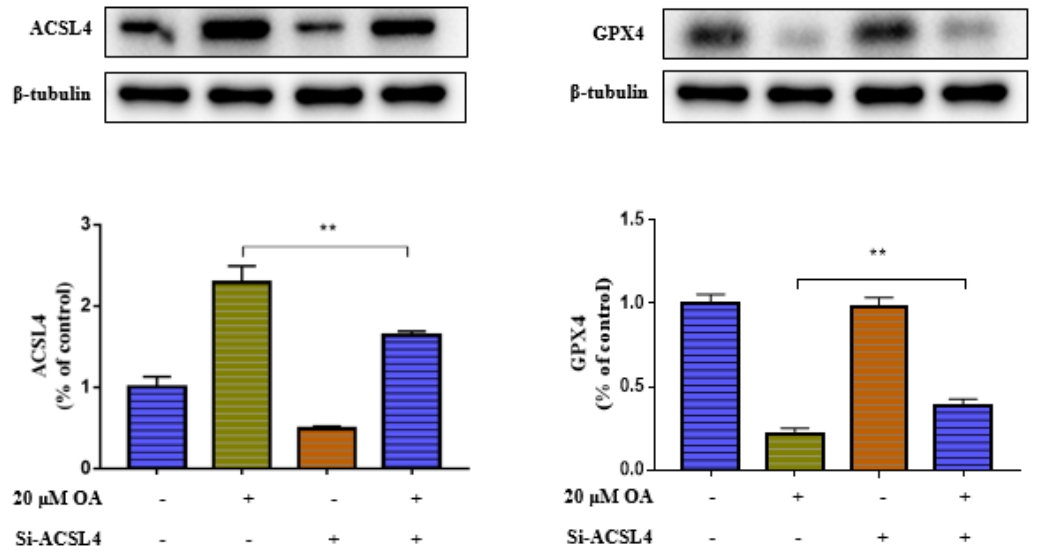

\section{Figure 6}

Silencing of ACSL4 decreases the sensitivity of Hela cells to OA. (A) ShRNA interference was successful and ACSL4 is silenced. (B) ACSL4 silencing decreases the viability of the Hela cells. (C) Representative images of EdU and Hoechst33342. (D) ACSL4 silencing reduces the proliferation efficiency of the Hela cells. (E) Detection of ROS by flow cytometry. (F) OA increased the expression of ACSL4. (G) OA 
decreased the expression of GPX4. Compared with the control group, ${ }^{*} P<0.05,{ }^{*} P<0.01$. Data are expressed as mean $\pm S D(n=3)$.

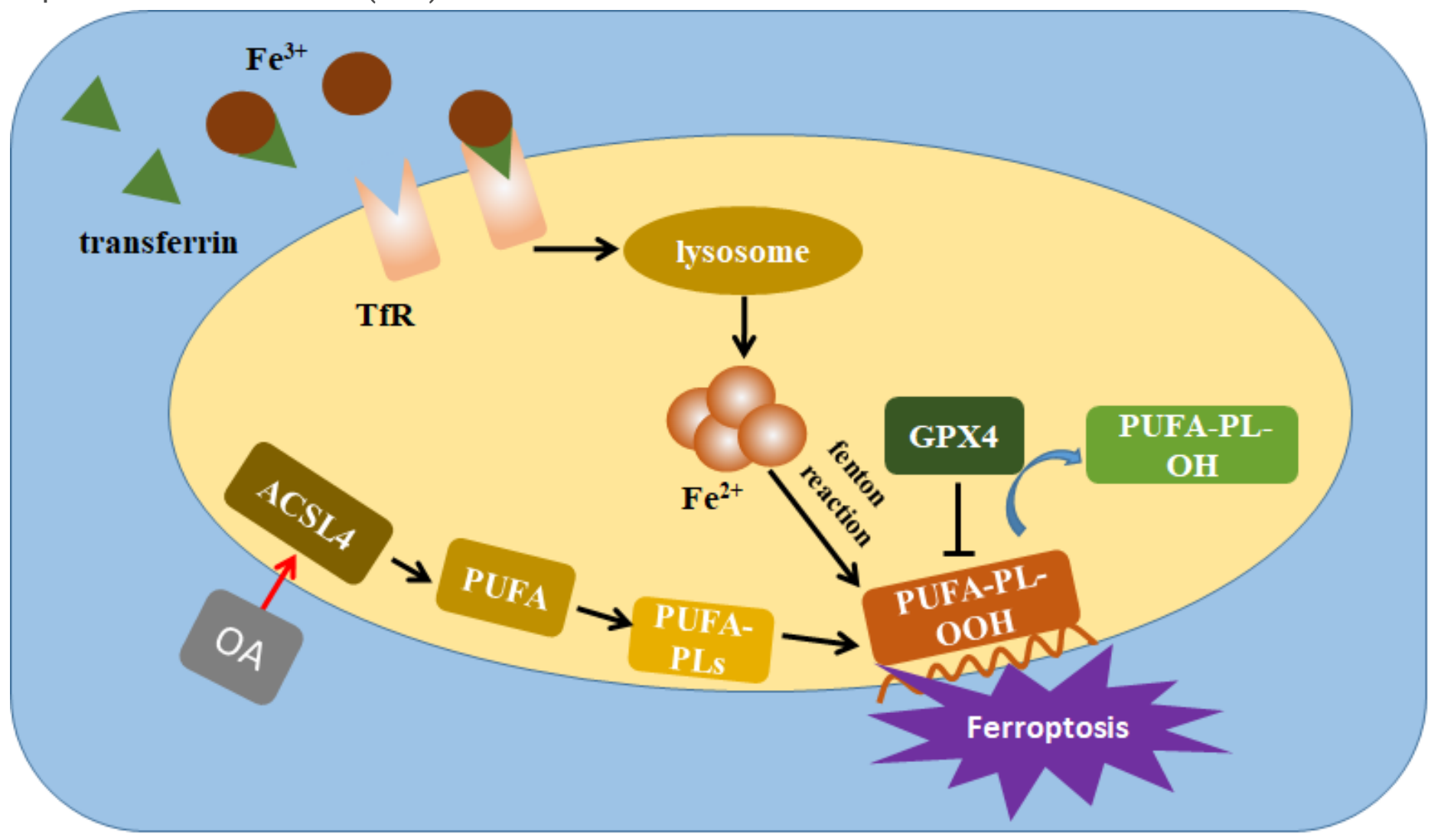

Figure 7

The mechanism of OA against cervical cancer. ACSL4, acyl-CoA synthase long-chain 4; PUFA, polyunsaturated fatty acid; PUFA-PLs, polyunsaturated fatty acid phospholipids; PUFA-PL-OOH, lipid peroxide; GPX4, glutathione peroxidase; TfR, transferrin receptor. 